\title{
Activation of the JNK pathway is important for cardiomyocyte death in response to simulated ischemia
}

\author{
Huaping $\mathrm{He}^{1}$, Hai-Ling $\mathrm{Li}^{2}$, Anning $\mathrm{Lin}^{3}$ and \\ Roberta A Gottlieb*,1 \\ ${ }^{1}$ Molecular and Experimental Medicine, The Scripps Research Institute, \\ La Jolla, California, USA \\ 2 Research Service, Veterans Administration Medical Center, San Diego, \\ California, USA \\ ${ }^{3}$ Department of Pathology, University of Alabama at Birmingham, Birmingham, \\ Alabama, USA \\ * corresponding author: Roberta A Gottlieb, Department of Molecular and \\ Experimental Medicine, The Scripps Research Institute, La Jolla, California, \\ CA 92037, USA. tel: (858)784-7929; fax: (858)784-7981; \\ email: robbieg@scripps.edu
}

Received 6.4.99; revised 2.7.99; accepted 30.7.99

Edited by J Reed

\begin{abstract}
Multiple signaling pathways, including the c-Jun N-terminal kinase (JNK) pathway, are activated in myocardial ischemia and reperfusion (MI/R) and correlate with cell death. However, the role of the JNK pathway in MI/R-induced cell death is poorly understood. In a rabbit model, we found that ischemia followed by reperfusion resulted in JNK activation which could be detected in cytosol as well as in mitochondria. To address the functional role of the JNK activation, we examined the consequences of blockade of JNK activation in isolated cardiomyocytes under conditions of simulated ischemia. The JNK activity was stimulated $\sim$ sixfold by simulated ischemia and reperfusion (simulated MI). When a dominant negative mutant of JNK kinase-2 (dnJNKK2), an upstream regulator of JNK, and JNK-interacting protein-1 (JIP-1) were expressed in myocytes by recombinant adenovirus, the activation of JNK by simulated MI was reduced $53 \%$. Furthermore, the TNF $\alpha$ activated JNK activity in H9c2 cells was completely abolished by dnJNKK2 and JIP-1. In correlation, when dnJNKK2 and JIP1 were expressed in cardiomyocytes, both constructs significantly reduced cell death after simulated MI compared to vector controls. We conclude that activation of the JNK cascade is important for cardiomyocyte death in response to simulated ischemia.
\end{abstract}

Keywords: cardiomyocytes; cell death; JNK; ischemia; adenovirus

Abbreviations: Adv, adenovirus; JIP-1, JNK-interacting protein-1; JNK, c-jun N-terminal kinase; JNKK2, JNK kinase 2; MI/R, myocardial ischemia and reperfusion

\section{Introduction}

Myocardial ischemia or ischemia followed by reperfusion ( $\mathrm{Ml}$ or $\mathrm{MI} / \mathrm{R}$ ) is known to induce cardiomyocyte death that contributes to heart failure. It has been recently recognized at the molecular level that ischemia/reperfusion-induced cell death involves activation of the c-jun $\mathrm{N}$-terminal kinase (JNK) pathway. ${ }^{1-3}$ In vitro, JNK has been shown to respond to a variety of stress signals including heat shock, ischemia, osmotic change, and inflammatory cytokines, ${ }^{4,5}$ and therefore is also named stress-activated protein kinase (SAPK). However, the functional role of JNK activation in stress-induced cell death is not fully understood. In fact, it remains controversial as to whether JNK activation represents a cellular protective mechanism or simply a signal for programmed cell death (apoptosis). For example, Yue et $a l^{6}$ showed that reduction of JNK activation by carvedilol correlated with a reduced number of apoptotic cardiomyocytes after myocardial ischemia and reperfusion, whereas in NIH3T3 fibroblasts the inactivation of JNK increased TNF $\alpha$-induced apoptosis. ${ }^{7}$ Discrepancies among these observations could be due to different roles of the JNK cascade in various cell types responding to differing stimuli, or due to the technical difficulty in dissecting one specific pathway from multiple cascades involved in the cellular response to stress.

JNK is regulated by its kinase, JNKK1 and 2. In contrast to JNKK1, JNKK2 only activates JNK, not p38. ${ }^{8-10}$ In addition, JNKK2 is expressed at a significant level in the heart. ${ }^{10} \mathrm{JNK}$ is also regulated by a recently identified cytosolic factor, JNK interacting protein-1 (JIP-1), which inhibits JNK from translocating into the nucleus. ${ }^{11}$ In the present study, we employed JIP-1 and a dominant negative mutant of JNKK2 (dnJNKK2) to interfere with activation of JNK in order to study the role of the JNK signaling pathway in cardiomyocytes under simulated ischemia conditions. Our data show that ischemia and reperfusion lead to JNK activation in cytosol and mitochondria and that the blockade of its activation by dnJNKK2 or by JIP-1 reduces cell death in isolated cardiomyocytes subjected to simulated ischemia.

\section{Results}

\section{Activation of JNK accompanies reperfusion after ischemia}

We used the rabbit open chest model to examine JNK activation in the heart in response to ischemia and reperfusion. Anesthetized ventilated rabbits were subjected to coronary artery ligation for $30 \mathrm{~min}$ followed by reperfusion for $15 \mathrm{~min}$. Myocardial tissue was harvested 
from ischemic and normally perfused regions of the left ventricle and cytosolic extracts were prepared, from which JNK activity was measured. A representative assay of JNK kinase activity is shown in Figure $1 \mathrm{~A}$ and the quantitative analysis of multiple samples is summarized in Figure $1 \mathrm{~B}$. We found a threefold increase in JNK activity in the ischemic and reperfused region when compared to the normally perfused tissue (Figure 1A), consistent with the findings of others. ${ }^{2,3}$ Western blot analysis demonstrated recovery of similar amounts of JNK in all samples (Figure $1 \mathrm{~A}$, bottom panel). In addition, in two animals in which tissue was recovered before reperfusion, activation of JNK was undetectable (Figure 1), suggesting it is closely tied to reperfusion. We examined cytosol and mitochondria for JNK activation and found that JNK activity was also significantly increased in the mitochondria after ischemia and reperfusion (Figure 1). These results implicated activation of the JNK pathway in ischemia/reperfusion injury but did not prove a causal role for JNK. To address this question, we turned to the isolated cardiomyocyte model and the use of adenovirus-mediated gene transfer of constructs intended to interfere with activation of JNK.

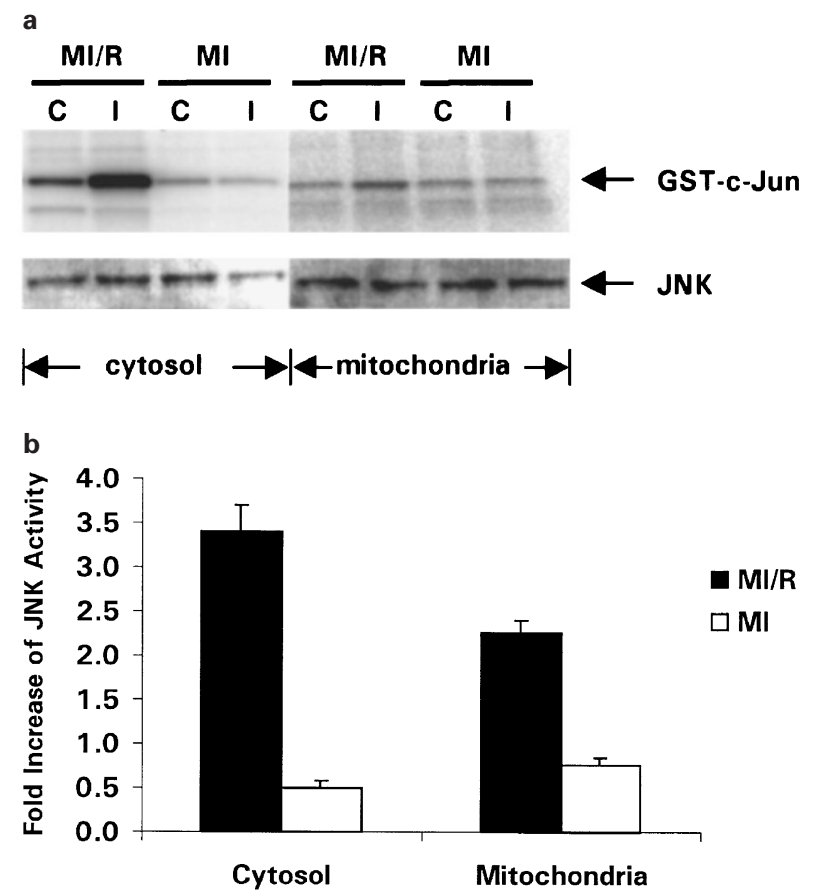

Figure 1 Ischemia/reperfusion results in activation of JNK in cytosol and mitochondria. Rabbits were subjected to ischemia and reperfusion (MI/R) or ischemia alone (MI), and cardiac tissue from the area-at-risk and normal left ventricle were homogenized, and cytosol and mitochondria were prepared. (A) A representative experiment showing phosphorylation of GST-C-Jun by immunoprecipitated JNK (top panel) and the Western blot of the immunoprecipitated JNK (bottom panel) from cytosol and mitochondria. ' $\mathrm{C}$ and ' $I$ ' represent tissue from control and ischemic region, respectively; (B) Summary of JNK assays in cytosol and mitochondria from myocardium after ischemia/reperfusion. The JNK activity is normalized to the amount of JNK as determined by Western blot. Activation is reported as fold increase over control. Data represent three ischemic and reperfused hearts and two ischemic-only hearts

\section{Activation of JNK by simulated MI or TNF $\alpha$ is inhibited by dnJNKK2 and JIP-1}

To examine the role of $\mathrm{JNK}$, we used isolated adult rabbit cardiomyocytes subjected to simulated $\mathrm{Ml}$ that included treatment with metabolic inhibitors, $1 \mathrm{mM} \mathrm{NaCN}$ and $20 \mathrm{mM}$ 2-deoxyglucose, for 40 min followed by 'reperfusion' with fresh media. First, we found that the simulated MI conditions (40 min of Ml and 20 min of R) stimulated JNK activity sixfold (Figure 2A). However, after dnJNKK2 or JIP1 were expressed in the cardiomyocytes by recombinant adenovirus for $24 \mathrm{~h}$ prior to the simulated $\mathrm{MI}$, the JNK activation by simulated MI was reduced by $53 \%$ (Figure $2 \mathrm{~A}$ ). The inhibitory effect of dnJNKK2 and JIP1 on JNK was further confirmed in H9c2 cells in which JNK activation by TNF $\alpha$ was abolished by dnJNKK2 and JIP1 (Figure 2B).

Since p38 MAPK cascade is often involved in stress response of cells, we examined the effect of simulated MI

a

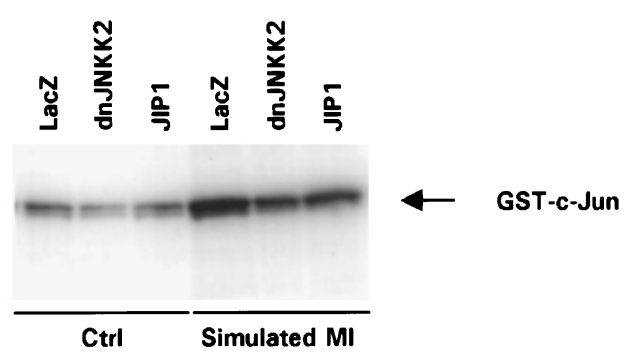

b
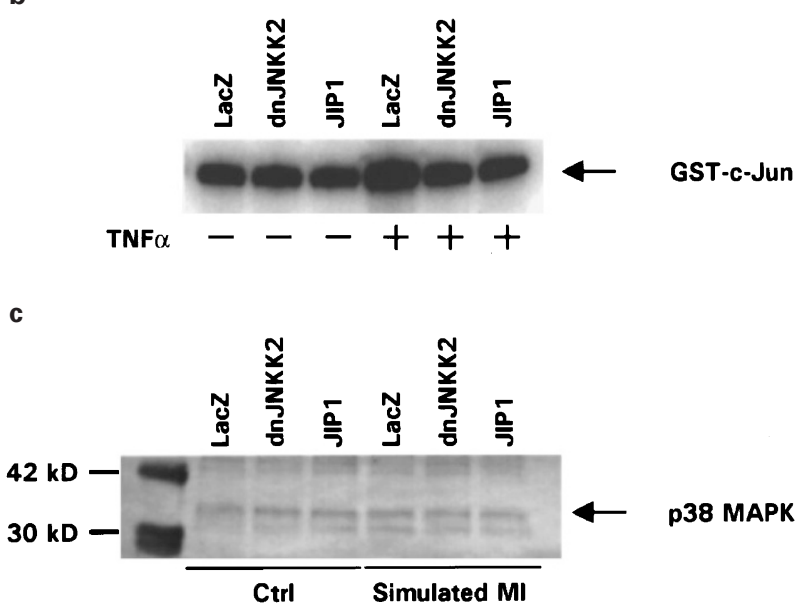

Figure 2 Characterization of JNK and p38 MAPKs in cardiomyocytes or H9c2 cells and effect of dnJNKK2 and JIP1 on JNK activity. (A) Cardiomyocytes were infected by adenovirus constructs expressing the indicated genes for $24 \mathrm{~h}$ before subjected to simulated $\mathrm{Ml}$ - metabolic inhibition for $40 \mathrm{~min}$ and recovery for $20 \mathrm{~min}$. A representative experiment showing effect of adenovirus constructs on phosphorylation of GST-C-Jun by immunoprecipitated JNK. (B) Cardiac myoblast cells, H9c2, were infected by the indicated adenoviruses for $24 \mathrm{~h}$ and then treated with (+) or without (-) TNF $\alpha$ for $30 \mathrm{~min}$ at a final concentration of $50 \mathrm{ng} / \mathrm{ml}$. A representative experiment showing effect of adenovirus constructs on phosphorylation of GST-c-Jun by immunoprecipitated JNK. (C) Phosphorylation of p38 MAPK was characterized by Western blot using a phospho-specific p38 polyclonal antibody (New England Biolab) following immunoprecipitation of total p38 with a p38 antibody (Santa Cruz Biotechnology) 
on this MAPK as well. $300 \mu \mathrm{g}$ of lysate protein was immunoprecipitated for total p38 MAPK (see Materials and Methods) followed by Western blot using phosphospecific p38 MAPK antibody to detect the activated p38 MAPK. As shown in Figure $2 \mathrm{C}$, simulated $\mathrm{Ml}$ had no significant effect on p38 MAPK, nor did dnJNKK2 or JIP1 have any effect on the basal activity of p38 MAPK.

\section{Inhibition of JNK activation correlated with reduction of cell death}

To examine the effect of blockade of JNK activation by simulated $\mathrm{Ml}$, we infected cardiomyocytes with Adv/ dnJNKK2, Adv/JIP-1, or $\operatorname{Adv} / S R(-)$ for $24 \mathrm{~h}$, then subjected the cells to $40 \mathrm{~min}$ metabolic inhibition and $3 \mathrm{~h}$ recovery. Viability was scored on the basis of preservation of rod-shaped morphology as previously described $^{12}$ and depicted as an example in Figure $3 \mathrm{~A}$. Results are expressed as per cent viable relative to control (set at 100\%). We found that adenovirus infection

a
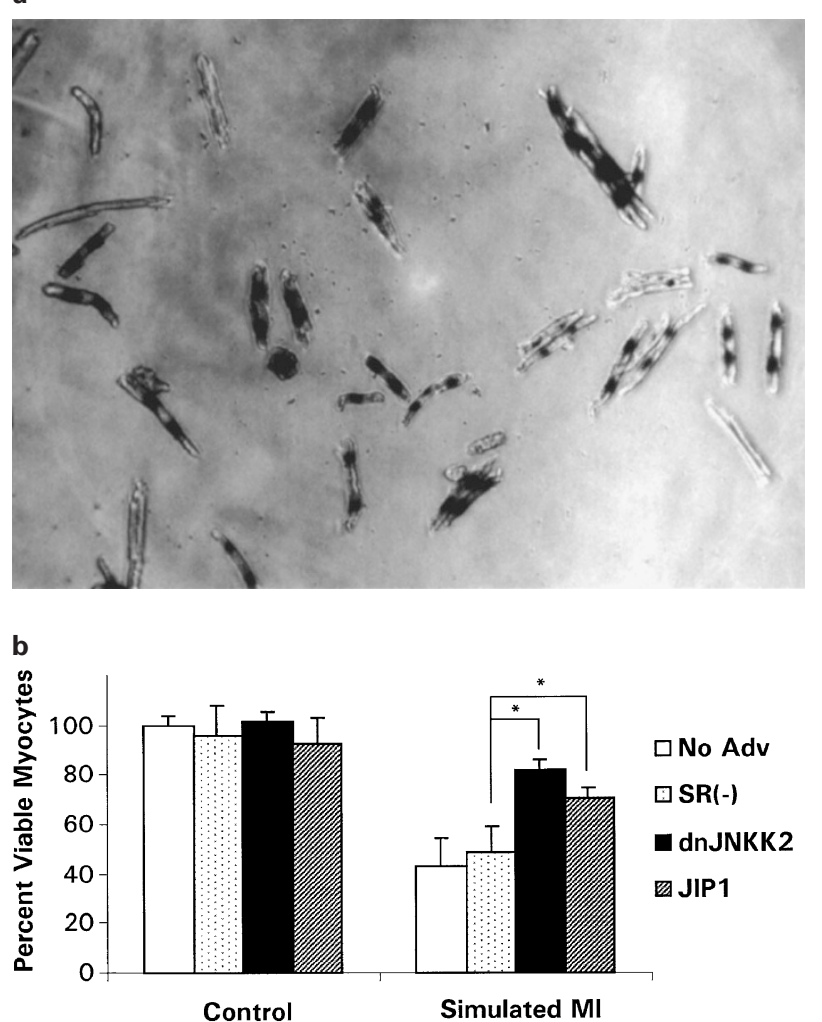

Figure 3 Effect of adenovirus constructs on cardiomyocyte survival after simulated ischemia. (A) Adult cardiomyocytes were infected by adenovirus expressing nuclear-localized LacZ for $24 \mathrm{~h}$ followed by staining for nuclear $\beta$ galactosidase activity. The picture also depicts the morphological criteria, rod vs round shape, for viability assays (see below); (B) Cardiomyocytes were infected with the indicated constructs for $24 \mathrm{~h}$ before subjected to simulated ischemia (simulated $\mathrm{MI}$ ). Results are reported as the per cent viable relative to uninfected and untreated control (set to $=100 \%$ ). Data represent the mean and standard deviation of three independent experiments. *Indicates statistical significance $(P<0.05)$ when compared to vector $(\mathrm{SR}(-))$ with the control vector did not have a significant effect on cell viability, nor did it affect cell death in response to simulated MI. However, both Adv/dnJNKK2 and Adv/JIP-1 were able to confer significant protection against metabolic inhibition and recovery (Figure $3 \mathrm{~B}$ ). These results demonstrate that activation of the JNK pathway is necessary for the induction of cell death in response to simulated ischemia.

\section{Discussion}

Previously others have shown that JNK is activated in response to ischemia/reperfusion, and that JNK activation could be detected within minutes after the onset of reperfusion. ${ }^{1-3}$ We also found JNK activation to accompany reperfusion in the rabbit heart. These findings suggest a role for JNK in the cell death response, but do not prove causality. In a variety of cell culture systems, JNK has been implicated in the cell death pathway; several mechanisms have been proposed. JNK has been shown to phosphorylate Bcl-2 which may result in loss of its antiapoptotic function. ${ }^{13}$ Some findings support a role for $\mathrm{Bcl}-2$ in cardioprotection. ${ }^{14-16}$ The JNK pathway activated by cell stress has also been shown to upregulate Fas ligand expression in Jurkat cells, leading to cell death via the Fas pathway. ${ }^{17}$ Inhibition of SEK1, an upstream regulator of JNK, blocked apoptosis in the neuronal cell line HN33. ${ }^{18}$ Of particular interest is the report by Yue et $a{ }^{6}{ }^{6}$ showing that reduction of JNK activation by carvedilol correlated with reduction of cardiomyocyte apoptosis in rats subjected to experimental ischemia and reperfusion. However, JNK may play a protective role in some settings such as TNF $\alpha$-induced apoptosis. ${ }^{7}$

We observed that JNK activity is increased in the mitochondria after ischemia and reperfusion. Mitochondria play a key role in regulating apoptosis through the release of cytochrome $c$ which serves as a cofactor for caspase activation, ${ }^{19}$ and also in regulating the balance of carnitine and palmitoylcarnitine; we have recently reported that carnitine inhibits caspases, while palmitoylcarnitine increases caspase activity (Mutomba et al, submitted). Bcl2 has been suggested to be one target of JNK. ${ }^{13}$ The association of JNK with the mitochondria following cellular stress is intriguing and leads us to speculate that phosphorylation of a mitochondrial target by JNK may play a role in regulating apoptosis.

The role of JNK was further examined in isolated cardiomyocytes subjected to metabolic inhibition (simulated $\mathrm{MI}$ ) which was previously shown to induce cardiomyocyte death. ${ }^{12}$ In the present study, we found that the JNK MAPK cascade was substantially activated by simulated $\mathrm{MI}$, which supports the notion that simulated $\mathrm{MI}$ in vitro induces cell death through similar stress kinase pathways and, therefore, provides a valid model system to study the molecular mechanisms underlying ischemiainduced cell death. Interestingly, p38 MAPK, that has been reported to respond to apoptotic signals in a variety of systems, was not significantly affected by simulated MI.

Next we specifically blocked the JNK activation by simulated $\mathrm{Ml}$ and examined the consequent effects on cell death. We chose JNKK2 because unlike JNKK1, 
JNKK2 is specific for phosphorylation of JNK without affecting p38/MAPK. ${ }^{9,10}$ In addition, JNKK2 is expressed at high levels in the heart. ${ }^{10}$ We found that dnJNKK2 and JIP1 partially blocked JNK activation by simulated MI, although in cardiac myoblast cells, H9c2, the constructs were highly effective in abolishing TNF $\alpha$-induced JNK activation. We speculate that the partial inhibition in myocytes might be due to the less efficient gene transfer that resulted in uninfected cells which contributed to uninhibited basal JNK-activity. Consistent with this, the protection of cells by dnJNKK2 or JIP1 failed to reach $100 \%$. Alternatively, the JNK pathway may have both protective and pro-apoptotic effects; in this experimental system, the pro-apoptotic effects appear to predominate.

Taken together, these results demonstrate an important role for activation of the JNK pathway in the induction of cell death following simulated ischemia. These results further suggest that inhibition of the JNK pathway at the time of myocardial reperfusion would reduce cell death.

\section{Materials and Methods}

\section{Open-chest ischemia model}

All animal procedures were approved by the animal care committee of the Veterans Affairs Medical Center. New Zealand white rabbits (2.5$3.5 \mathrm{~kg}$ ) were anesthetized with intravenous pentobarbital, then were intubated and ventilated. The heart was exposed through a midline thoracotomy and a suture ligature passed around the anterior branch of the left coronary artery. Ischemia was induced by snare occlusion and verified by recognition of blanching and abnormal wall motion. After 40 min of ischemia the snare was released and reperfusion maintained for $15 \mathrm{~min}$. The heart was then rapidly excised, hung in a Langendorff perfusion mode, the coronary bed flushed with lactated Ringer's solution. At this point, the ligature was snare occluded again and monastral blue dye was infused to mark the region of normal perfusion.

\section{Preparation of cytosol and mitochondria from heart tissue}

Hearts were removed from the perfusion device and transmura myocardial samples from normal and ischemic areas were isolated using monastral blue dye perfusion as a guide. All subsequent steps were performed at $4^{\circ} \mathrm{C}$ or on ice. Tissue was minced in pre-chilled homogenization buffer ( $225 \mathrm{mM}$ mannitol, $75 \mathrm{mM}$ sucrose, $50 \mathrm{mM}$ EDTA, $20 \mathrm{mM}$ HEPES, pH 7.4) then disrupted using a Polytron at speed 2 for $8 \mathrm{~s}$. Tissue fragments, unbroken cells, and nuclei were removed by sedimentation at $450 \mathrm{~g}$ for $10 \mathrm{~min}$. The supernatant was decanted through gauze and mitochondria were sedimented at $10000 \times g$ for $10 \mathrm{~min}$. The pellet was gently rinsed with homogenization buffer to remove the fluffy overlayer, then resuspended in homogenization buffer followed by centrifugation $(10000 \times g)$. The wash was repeated one more time. The postmitochondrial supernatant, referred to in the text as cytosol, were aliquotted and stored at $-70^{\circ} \mathrm{C}$ until use.

\section{Measurement of JNK and p38 activity}

The JNK activity was measured in immunoprecipitation-coupled kinase assay. The lysis of cells, immunoprecipitation of JNK and subsequent JNK assay followed a protocol as described. ${ }^{8}$ Cytosol
(100 $\mu \mathrm{g}$ protein) and mitochondria (45 $\mu \mathrm{g}$ protein) from ischemic and normal regions of the left ventricle, cardiomyocytes (300 $\mu$ g protein) or $\mathrm{H} 9 \mathrm{c} 2$ cells (100 $\mu \mathrm{g}$ protein) were dissolved in lysis buffer ( $1 \%$ Triton X$100,50 \mathrm{mM}$ Tris, pH 8.0, $150 \mathrm{mM} \mathrm{NaCl}, 100 \mu \mathrm{g} / \mathrm{ml} \mathrm{PMSF}, 1 \mu \mathrm{g} / \mathrm{ml}$ aprotinin). JNK was immunoprecipitated by incubation with $2 \mu \mathrm{g}$ of rabbit polyclonal antibody against human JNK (Santa Cruz Biotechnology, Santa Cruz, CA, USA) followed by incubation with immobilized Protein $\mathrm{G}$ (Pierce). Kinase assay was initiated by adding $1 \mu \mathrm{g}$ of GST-C-jun in $25 \mu \mathrm{l}$ of kinase buffer (20 mM HEPES, pH 7.6, $20 \mathrm{mM} \beta$-glycerolphosphate, $10 \mathrm{mM} \mathrm{p}$-nitrophenylphosphate, $10 \mathrm{mM}$ $\mathrm{MgCl}_{2}, 1 \mathrm{mM}$ DTT, $50 \mu \mathrm{M}$ sodium orthovanadate, $10 \mu \mathrm{M}$ ATP and $10 \mu \mathrm{Ci}$ of $\left.\left[\gamma_{-}{ }^{32} \mathrm{P}\right] \mathrm{ATP}\right)$ and incubated at $30^{\circ} \mathrm{C}$ for $20 \mathrm{~min}$.

The p38 MAPK activity was measured by Western blot using phospho-specific antibody. In brief, $300 \mu \mathrm{g}$ protein was immunoprecipitated (see above Materials and methods) for total p38 MAPK with a p38 polyclonal antibody (Santa Cruz Biotechnology, Santa Cruz, CA, USA). The purified p38 was resolved on a SDS-PAGE, transferred to nitrocellulose membrane and blotted with phospho-specific p38 antibody (New England Biolab, MA, USA).

\section{Plasmids and adenovirus vector}

A dominant negative mutant of human JNKK2 (dnJNKK2) was created by site-directed mutagenesis (Lys 149 was replaced by Met using Chameleon ${ }^{(i x}$ double-stranded site-directed mutagenesis kit (Stratagene)) and confirmed by sequencing. JIP-1 was a generous gift from Roger J Davis, University of Massachusetts, Worcester, MA, USA. Adenovirus shuttle vector, pACCMV.pLpA, or SR $(-)$ in abbreviation, was kindly provided by Robert D Girard, University of Texas Southwestern Medical Center, Dallas, TX, USA. The adenovirus plasmid, pJM17, was a generous gift from Frank L Graham, McMaster University, Canada. GST-c-jun expression plasmid was kindly provided by Michael Karin, University of California San Diego, CA, USA.

\section{Construction of recombinant adenovirus}

Cloning of transgenes into a replication-deficient human Adv (type 5) followed a protocol described by Becker et al. ${ }^{20}$ In brief, dnJNKK2 and JIP-1 were first cloned between CMV enhancer/promoter and SV40 polyadenylation signal sequence of shuttle vector $\mathrm{SR}(-)$. The shuttle constructs were then cotransfected with pJMI7 into HEK293 cells. Adv containing the gene of interest was generated through homologous recombination between the two vectors in 293 cells. $^{20}$ In addition, Advs expressing an empty shuttle vector $\mathrm{SR}(-)(\mathrm{Adv} / \mathrm{SR}(-))$ or LacZ (Adv/LacZ) were used in experiments as controls which produced essentially the same results as controls with no viral infection (data not shown). The Advs were verified by either Western blot or PCR (data not shown) and subsequently propagated, purified by $\mathrm{CsCl}$ gradient centrifugation and plaque-titered as described. ${ }^{20}$

\section{Isolation and adenoviral infection of rabbit cardiac myocytes and H9c2 cells}

All studies were approved by the Animal Committee of The Scripps Research Institute. The preparation of adult rabbit ventricular myocytes followed a protocol described previously ${ }^{12}$ except that the final resuspension of myocytes was in medium M199. For adenoviral infection, adenovirus was added to myocytes at multiplicity of infection (MOI) of 100 and incubated for $1 \mathrm{~h}$ at room temperature on a slow rocking platform. Following infection, media were replenished and myocytes were incubated in a humidified incubator $\left(37^{\circ} \mathrm{C}, 5 \% \mathrm{CO}_{2}\right)$ for $24 \mathrm{~h}$ before use in simulated ischemia experiments. 
The H9c2 rat embryonic heart cell line was infected by adenovirus constructs at $\mathrm{MOI}$ of 100 for $1 \mathrm{~h}$ followed by $24 \mathrm{~h}$ incubation. Cells were treated with human tumor necrosis factor $\alpha$ (TNF $\alpha)$ for 30 min at final concentration of $50 \mathrm{ng} / \mathrm{ml}$ prior to lysis for JNK activity assays.

\section{Metabolic inhibition and viability assays}

Rabbit cardiac myocytes were infected with Adv for $24 \mathrm{~h}$ before being subjected to metabolic inhibition. The metabolic inhibition protocol was essentially identical to the previous report ${ }^{12}$ except that the incubation time for metabolic inhibition and recovery was $40 \mathrm{~min}$ and $3 \mathrm{~h}$, respectively. Myocytes were transferred to glass slides and fixed with $1.25 \%$ formaldehyde. The viability of myocytes following metabolic inhibition and recovery was scored based on the previously established criteria, i.e., rod-shaped (viable) cells vs round-shaped (non-viable) cells (Figure 3A). This method has been compared to TUNEL assays ${ }^{12}$ and established to be a reliable measure of viability. Under light microscope, a total of four fields were randomly selected, in which a minimum of 300 cells were counted by observers blinded to the identity of the conditions. The percent viable myocytes is defined as the percentage of rod-shaped cells in the total number of cells.

\section{Statistical analysis of data}

Data are expressed as mean \pm standard deviation (S.D.). One-way ANOVA analysis was used to examine the statistical difference between control (SR $(-)$ or LacZ), dnJNKK2 and JIP-1 in the same experimental group. Statistical significance is defined as $P<0.05$.

\section{Acknowledgements}

This work was supported by NIH/NHLBI R01 HL60590, HL61518, and a grant from Novartis. The authors gratefully acknowledge the expertise and assistance of Dr. Robert Engler in experiments involving the openchest ischemia/reperfusion model. The authors also gratefully acknowledge the helpful discussion of Dr. Zhenguo Wu and technical assistance of Yi Liu and Sharon Lu.

\section{References}

1. Bogoyevitch MA, Gillespie-Brown J, Ketterman AJ, Fuller SJ, Ben-Levy R, Ashworth A, Marshall CJ and Sugden PH (1996) Stimulation of the stressactivated mitogen-activated protein kinase subfamilies in perfused heart: p38/ RK mitogen-activated protein kinases and c-Jun N-terminal kinases are activated by ischemia/reperfusion. Circ. Res. 79: 162-173

2. Knight RJ and Buxton DB (1996) Stimulation of C-Jun kinase and mitogenactivated protein kinase by ischemia and reperfusion in the perfused rat heart. Biochem. Biophys. Res. Commun. 218: 83-88

3. Yin T, Sandhu G, Wolfgang CD, Burrier A, Webb RL, RigelDF, Hai TandWhelan J (1997) Tissue-specific pattern of stress kinase activation in ischemic/reperfused heart and kidney. J. Biol. Chem. 272: 19943-19950
4. Bogoyevitch MA, Ketterman AJ and Sugden PH (1995) Cellular stresses differentially activate c-Jun $\mathrm{N}$-terminal protein kinases and extracellular signalregulated protein kinases in cultured ventricular myocytes. J. Biol. Chem. 270: 29710-29717

5. Clerk A, Michael A and Sugden PH (1998) Stimulation of multiple mitogenactivated protein kinase sub-families by oxidative stress and phosphorylation of the small heat shock protein, HSP25/27, in neonatal ventricular myocytes. Biochem. J. 333: $581-589$

6. Yue T-L, Ma X-L, Wang X, Romanic AM, Liu G-L, Louden C, Gu J-L, Kumar S, Poste G, Ruffolo Jr RR and Feuerstein GZ (1998) Possible involvement of stressactivated protein kinase signaling pathway and Fas receptor expression in prevention of ischemia/reperfusion-induced cardiomyocyte apoptosis by carvedilol. Circ. Res. 82: 166-174

7. Roulston A, Reinhard C, Amiri P and Williams LT (1998) Early activation of c-Jun $\mathrm{N}$-terminal kinase and p38 kinase regulate cell survival in response to tumor necrosis factor $\alpha$. J. Biol. Chem. 273: 10232-10239

8. Lin A, Minden A, Martinetto H, Claret FX, Lange-Carter CA, Mercurio F, Johnson $\mathrm{GL}$ and Karin M (1995) Identification of a dual specificity kinase that activates the Jun kinases and p38-Mpk2. Science 268: 286-290

9. Lu X, Nemoto S and Lin A (1997) Identification of C-Jun $\mathrm{NH}_{2}$-terminal protein kinase (JNK)-activating kinase 2 as an activator of JNK butnotp38. J. Biol. Chem. 272: $24751-24754$

10. WuZ, Wu J, Jacinto E and Karin M (1997) Molecular cloning and characterization of human JNKK2, a novel Jun $\mathrm{NH}_{2}$-terminal kinase-specific kinase. Mol. Cell Biol. 17: 7407-7416

11. Dickens M, Rogers JS, Cavanagh J, Raitano A, Xia Z, Halpern JR, Greenberg ME, Sawyers CL and Davis RJ (1997) A cytoplasmic inhibitor of the JNK signal transduction pathway. Science 277: 693-696

12. Gottlieb RA, Gruol DL, Zhu JY and Engler RL (1996) Preconditioning in rabbit cardiomyocytes: Role of $\mathrm{pH}$, vacuolar proton ATPase, and apoptosis. J. Clin. Invest. 97: 2391-2398

13. Maundrell K, Antonsson B, Magnenat E, Camps M, Muda M, Chabert C, Gillieron C, Boschert U, VialKnecht E, Martinou JC and Arkinstall S (1997) Bcl-2 undergoes phosphorylation by c-Jun $\mathrm{N}$-terminal kinase/stress-activated protein kinases in the presence of the constitutively active GTP-binding protein Rac1. J. Biol. Chem. 272: 25238-25242

14. Kirshenbaum LA and de Moissac D (1997) The bcl-2 gene product prevents programmed cell death of ventricular myocytes. Circ. 96: 1580-1585

15. Misao J, Hayakawa Y, Ohno M, Kato S, Fujiwara T and Fujiwara H (1996) Expression of bcl-2 protein, an inhibitor of apoptosis, and Bax, an accelerator of apoptosis, in ventricular myocytes of human hearts with myocardial infarction. Circ. 94: 1506-1512

16. Kirshenbaum LA (1998) Regulators of apoptosis in the heart: a matter of life and death. Can. J. Cardiol. 14: 457-460

17. Faris M, Kokot N, Latinis K, Kasibhatla S, Green DR, Koretzky GA and Nel A (1998) The c-Jun N-terminal kinase cascade plays a role in stress-induced apoptosis in Jurkat cells by up-regulating Fas ligand expression. J. Immunol. 160: $134-144$

18. Liu YF (1998) Expression of polyglutamine-expanded Huntingtin activates the SEK1-JNK pathway and induces apoptosis in a hippocampal neuronal cell line.J. Biol. Chem. 273: 28873-28877

19. Reed JC (1997) Cytochrome c: Can't live with it-can't live without it. Cell 91 $559-562$

20. Becker TC, Noel RJ, Coats WS, Gomez-Foix AM, Alarn T, Gerard RD and Newgard CB (1994) Use of adenovirus for metabolic engineering of mammalian cells. Methods in Cell Biology 43: 161-189 